\title{
Photometric constraints on microlens spectroscopy of EROS-BLG-2000-5
}

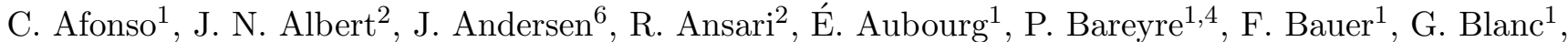

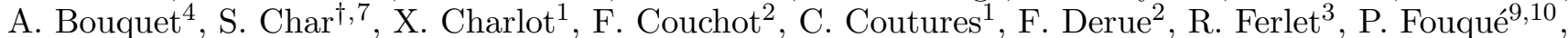
J. F. Glicenstein ${ }^{1}$, B. Goldman ${ }^{1}$, A. Gould ${ }^{8,4}$, D. Graff ${ }^{8,1}$, M. Gros ${ }^{1}$, J. Haïssinski ${ }^{2}$, J. C. Hamilton ${ }^{4}$, D. Hardin ${ }^{1}$, J. de Kat ${ }^{1}$, A. Kim ${ }^{4}$, T. Lasserre ${ }^{1}$, L. LeGuillou ${ }^{1}$, É. Lesquoy ${ }^{1,3}$, C. Loup ${ }^{3}$, C. Magneville ${ }^{1}$, B. Mansoux ${ }^{2}$, J. B. Marquette ${ }^{3}$, É. Maurice ${ }^{5}$, A. Milsztajn ${ }^{1}$, M. Moniez ${ }^{2}$, N. Palanque-Delabrouille ${ }^{1}$, O. Perdereau ${ }^{2}$, L. Prévot ${ }^{5}$, N. Regnault ${ }^{2}$, J. Rich ${ }^{1}$, M. Spiro ${ }^{1}$, A. Vidal-Madjar ${ }^{3}$, and L. Vigroux ${ }^{1}$, and S. Zylberajch ${ }^{1}$ (The EROS collaboration)

1 CEA, DSM, DAPNIA, Centre d'Études de Saclay, 91191 Gif-sur-Yvette Cedex, France

2 Laboratoire de l'Accélérateur Linéaire, IN2P3 CNRS, Université de Paris-Sud, 91405 Orsay Cedex, France

3 Institut d'Astrophysique de Paris, INSU CNRS, 98bis boulevard Arago, 75014 Paris, France

${ }^{4}$ Collège de France, Physique Corpusculaire et Cosmologie, IN2P3 CNRS, 11 Pl. M. Berthelot, 75231 Paris Cedex, France

5 Observatoire de Marseille, 2 Pl. Le Verrier, 13248 Marseille Cedex 04, France

6 Astronomical Observatory, Copenhagen University, Juliane Maries Vej 30, 2100 Copenhagen, Denmark

7 Universidad de la Serena, Facultad de Ciencias, Departamento de Fisica, Casilla 554, La Serena, Chile

8 Departments of Astronomy and Physics, Ohio State University, Columbus, OH 43210, USA

${ }^{9}$ Observatoire de Paris-Meudon DESPA, 92195 Meudon Cedex, France

10 European Southern Observatory, Casilla 19001, Santiago 19, Chile

Received 8 June 2001 / Accepted 8 August 2001

\begin{abstract}
We apply EROS photometric data to interpret previously published Keck and VLT spectra of the binary-microlens caustic-crossing event EROS-BLG-2000-5. We show that the VLT data imply that the outer $\sim 4 \%$ of the limb of the K3-giant source is strongly in emission in $\mathrm{H} \alpha$, in contradiction to available models of the photosphere. This conflict could be resolved if the integrated $\mathrm{H} \alpha$ emission from the chromosphere were equal to $8 \%$ of the integrated $\mathrm{H} \alpha$ absorption from the source as a whole. These conclusions regarding the extreme limb are almost completely model-independent. We also present a general method for using the photometric data to facilitate direct comparison between the atmospheric model and the spectroscopic data. While this method has some model-dependent features, it is fairly robust and can serve to guide the analysis of spectra while more detailed models of the lens geometry are being developed. In addition, we find that the color of the limb of the source (outer $5.5 \%$ by radius) is $\Delta(V-I) \sim 0.37$ redder than the source as a whole, so that it has the color of a M0 giant.
\end{abstract}

Key words. gravitational lensing - techniques: high angular resolution techniques: spectroscopic - stars: atmospheres

\section{Introduction}

Microlensing provides a potentially powerful probe of stellar atmospheres. If a lens caustic (region of formally infinite magnification) passes over the face of the source, then different parts of the atmosphere become highly magnified at different times. By combining a time series of photometric or spectroscopic observations, one can therefore hope to deconvolve the spatial structure of the atmosphere. For point lenses, the caustics are point-like, and therefore the probability of such a caustic crossing is small. However,

Send offprint requests to: J. F. Glicenstein,

e-mail: glicens@hep.saclay.cea.fr;

gould@astronomy. ohio-state.edu if the lens is a binary with components separated by of order the Einstein radius, the caustics form one to three concave polygons whose total length is of the order of the Einstein radius, and therefore the probability of a caustic crossing is much larger.

Intensive photometric observations of four such binary caustic crossing events have yielded limb-darkening measurements (Afonso et al. 2000; Albrow et al. 1999, 2000, 2001a). A more ambitious project would be to obtain a similar intensive series of spectroscopic measurements. Since light from the limb of the star originates higher in the atmosphere than light from the center, deconvolution of a set of spectral measurements effectively resolves the 
atmosphere as a function of height (Valls-Gabaud 1998; Heyrovský et al. 2001; Gaudi \& Gould 1999). Until the advent of microlensing, such spatially resolved spectra had been obtained only for the Sun.

The first spectra of a caustic-crossing event were obtained by Lennon et al. (1996). Alcock et al. (1997) found differences in the spectra of an extended source event as a function of time that were consistent with the predictions of models.

The binary caustic crossing event EROS-BLG-2000-5 has now yielded a major new breakthrough. This event was alerted by $\mathrm{EROS}^{1}$ on 5 May 2000. On 8 June 2000, $\mathrm{MPS}^{2}$ issued an anomaly alert, saying that a caustic crossing was in progress. Subsequent intensive observations by PLANET $^{3}$ allowed them to predict the time of the second caustic crossing and, very importantly, that this crossing would last an unusually long 4 days. This prediction permitted two groups to acquire spectra with large telescopes on several successive nights: Castro et al. (2001) obtained HIRES $(R \sim 40000)$ spectra using Keck on the last two nights of the crossing, while Albrow et al. (2001b) obtained low-resolution $(R \sim 1000)$ FORS1 spectra using VLT on all four nights. In addition, Albrow et al. (2001b) obtained a spectrum well before the caustic crossing, when the magnification of the source was approximately uniform.

To date, each group has published only the $\mathrm{H} \alpha$ lines, but both sets of spectra cover several thousand $\AA$, and could potentially yield a wealth of information about the $\mathrm{K} 3$ giant source.

Here we use EROS photometric observations of this event to aid in the interpretation of the spectral data that have been published. In Sect. 2, we present the EROS photometric data of the event. In Sect. 3, we present an essentially model-independent argument to show that the VLT data imply that the outer $\sim 4 \%$ of the limb of the source is very strongly in emission in $\mathrm{H} \alpha$. In Sect. 4, we develop a general method to apply photometric data of a caustic crossing to facilitate comparison between spectroscopic data and atmospheric models and apply this method to EROS-BLG-2000-5. In Sect. 5, we characterize the limits of our approach. In Sect. 6, we measure the color of the source limb by applying a slightly modified version of our approach to the EROS photometric data. Finally, in Sect. 7, we speculate about the possibility that the $\mathrm{H} \alpha$ emission detected from the limb of the source is actually due to the chromosphere, and quantify the strength of chromospheric emission required to explain the observations.

If the geometry of EROS-BLG-2000-5 were perfectly modelled, then there would be no need for the techniques introduced in this paper. However, the event is quite complex and difficult to model, so additional techniques are required to begin quantitative investigation of the

\footnotetext{
${ }^{1}$ http://www-dapnia.cea.fr/Spp/Experiences/EROS/ alertes.html

2 http://bustard.phys.nd.edu/MPS/index.html

3 http://thales.astro.rug.nl/ planet/
}

spectroscopic data while a better geometric model is being developed. Moreover, it is likely that in future events, one will face a qualitatively similar situation, and the methods presented in this paper will be applicable long before the event as a whole is analyzed at a satisfactory level.

\section{Photometric data}

EROS observations were carried out using the $1 \mathrm{~m}$ Marly telescope at La Silla, Chile, in two bands $V_{\mathrm{E}}$ and $I_{\mathrm{E}}$. EROS $V_{\mathrm{E}}$ is centered midway between Johnson $V$ and Cousins $R$, while EROS $I_{\mathrm{E}}$ is similar to Cousins $I$, but broader. The transformation between the Eros system $\left(V_{\mathrm{E}}, I_{\mathrm{E}}\right)$ and the standard Johnson-Cousins $(V, I)$ system was determined by observing Landolt (1992) standards near the galactic poles and Paczynski et al. (1999) secondary standards in the Baade window:

$V_{\mathrm{E}}=0.71 V+0.29 I+$ const.,

$I_{\mathrm{E}}=-0.02 \mathrm{~V}+1.02 I+$ const.

These equations taken together together imply $\Delta\left(V_{\mathrm{E}}-\right.$ $\left.I_{\mathrm{E}}\right)=0.73 \Delta(V-I)$. The first of Eq. (1) implies that the centroid of the $V_{\mathrm{E}}$ band is approximately $6300 \AA$. We therefore present mainly $V_{\mathrm{E}}$ data because its centroid is close to $\mathrm{H} \alpha$. EROS observations are normally carried out in survey mode, but owing to the importance of this event we devoted all available time to it during the caustic crossing. Generally, the weather was good, but on the final night there were intermittent clouds, and then finally we were clouded out entirely four hours before dawn.

The data were initially reduced on site using our standard PEIDA (Ansari et al. 1996) PSF-based software, but were then reprocessed using the ISIS (Alard 2000) image subtraction program. We then determined the zero point of the image-subtracted photometry by fitting for the mean offset between it and the PEIDA photometry. Figure 1 shows the naive magnification $A_{V}$, which we determine by dividing the measured flux by the baseline flux. The curve will be explained in Sect. 4, but from the data points alone, it is clear that the caustic crossing ended some time before $\mathrm{JD}^{\prime}=1733.6506$ : the light curve is almost perfectly flat commencing at this time and continuing for $58 \mathrm{~min}$, with a measured slope (combining information from both bands) of $\mathrm{d} \ln A / \mathrm{d} t=0.11 \pm 0.08 \mathrm{day}^{-1}$. (Here $\mathrm{JD}^{\prime}=\mathrm{JD}-2450000$.)

The crosses indicate the times of the Keck and VLT observations. Note that the VLT observations were all coincident with our photometric observations, while the Keck observations both took place about $2.2 \mathrm{hrs}$ after the end of our night.

\section{3. $\mathrm{H} \alpha$ in emission?}

The last VLT equivalent width $(E W)$ measurement, which was taken just before the source finally exited the caustic, is much lower than any of the other $E W$ measurements. As we will show a few paragraphs below, this can be interpreted as indicating strong $\mathrm{H} \alpha$ emission from the edge 


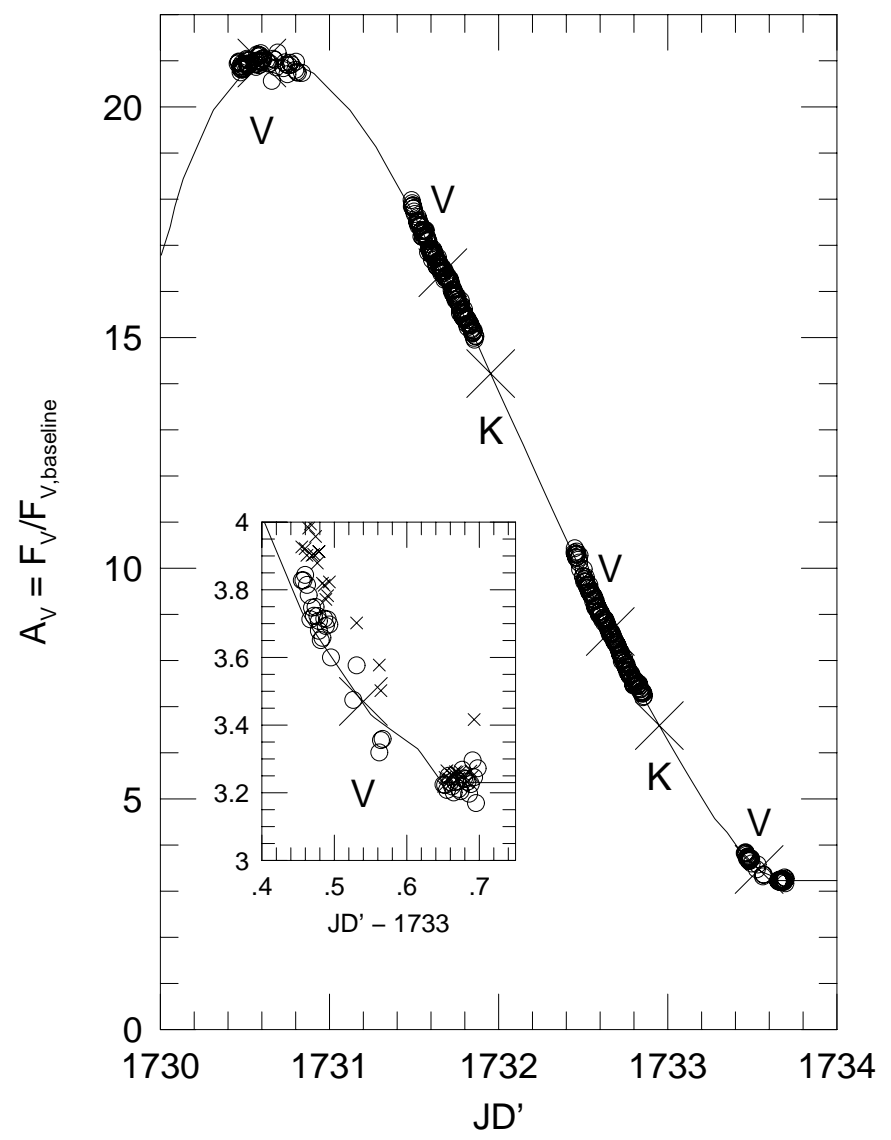

Fig. 1. Magnification $A_{V}$ of a K3 giant during the second caustic crossing of EROS-BLG-2000-5. Open circles show the fluxes in the EROS $V_{\mathrm{E}}$ band divided by the baseline flux (i.e., assuming no blending.) Large crosses represent the times of spectroscopic observations by Albrow et al. (2001b) at the VLT (V) and by Castro et al. (2001) at Keck (K). The curve is the projection of the simple quadratic model shown in Fig. 2 into (magnification,time) coordinates using the stellar-profile function $G(\eta)$ (Eq. (10)) derived from Fig. 1 of Albrow et al. (2001b). (Irregularities are due to the difficulty of transcribing the figure.) The insert also shows EROS $I_{\mathrm{E}}$ points (crosses) for the last night. From the two bands together, the slope of the magnification during the hour after the caustic exit at $\mathrm{JD}^{\prime}=1733.65$ is $\mathrm{d} \ln A / \mathrm{d} t=(0.11 \pm 0.08)$ day $^{-1}$. Here, $\mathrm{JD}^{\prime}=\mathrm{JD}-2450000$.

of the star. In order to quantify this conclusion, we must first derive some relations between the photometric and spectroscopic data.

Let $A(\boldsymbol{u})$ be the magnification field of the binary as a function of angular position $\boldsymbol{u}$ normalized to the Einstein radius, let $E(\boldsymbol{u})$ be the equivalent width of the $\mathrm{H} \alpha$ line, and let $S(\boldsymbol{u})$ be the source surface brightness in the neighborhood of this line. We will split the magnification field into two components $A(\boldsymbol{u})=A_{2}(\boldsymbol{u})+A_{3}(\boldsymbol{u})$ corresponding respectively to the two images whose magnification diverges (and then vanishes) at the caustic, and the three non-divergent images. The observed magnification is then,

$\bar{A}\left(\boldsymbol{u}_{\mathrm{c}}\right)=\frac{\int \mathrm{d}^{2} u A(\boldsymbol{u}) S(\boldsymbol{u})}{\int \mathrm{d}^{2} u S(\boldsymbol{u})}=A_{3}\left(\boldsymbol{u}_{\mathrm{c}}\right)+\overline{A_{2}}\left(\boldsymbol{u}_{\mathrm{c}}\right)$, where

$\overline{A_{2}}\left(\boldsymbol{u}_{\mathrm{c}}\right) \equiv \frac{\int \mathrm{d}^{2} u A_{2}(\boldsymbol{u}) S(\boldsymbol{u})}{F_{\mathrm{s}}}, \quad F_{\mathrm{s}} \equiv \int \mathrm{d}^{2} u S(\boldsymbol{u})$,

and where we have assumed that the non-divergent magnification field can be replaced by its value at the center of the source $\boldsymbol{u}_{\mathrm{c}}$. This is strictly true whenever this magnification field is either flat or has a uniform slope. We will discuss in Sect. 5 why we believe this to be an extremely good approximation in the present case.

Similarly, the observed $\mathrm{H} \alpha E W$ will be

$$
\begin{aligned}
\bar{E}\left(\boldsymbol{u}_{\mathrm{c}}\right) & =\frac{\int \mathrm{d}^{2} u A(\boldsymbol{u}) S(\boldsymbol{u}) E(\boldsymbol{u})}{\int \mathrm{d}^{2} u A(\boldsymbol{u}) S(\boldsymbol{u})} \\
& =\frac{A_{3}\left(\boldsymbol{u}_{\mathrm{c}}\right) E_{0}+\overline{A_{2}}\left(\boldsymbol{u}_{\mathrm{c}}\right) \overline{E_{2}}\left(\boldsymbol{u}_{\mathrm{c}}\right)}{A_{3}\left(\boldsymbol{u}_{\mathrm{c}}\right)+\overline{A_{2}}\left(\boldsymbol{u}_{\mathrm{c}}\right)},
\end{aligned}
$$

where, $E_{0}$ is the $E W$ of the unmagnified source, and

$\overline{E_{2}}\left(\boldsymbol{u}_{\mathrm{c}}\right) \equiv \frac{\int \mathrm{d}^{2} u A_{2}(\boldsymbol{u}) S(\boldsymbol{u}) E(\boldsymbol{u})}{F_{\mathrm{s}} \overline{A_{2}}\left(\boldsymbol{u}_{\mathrm{c}}\right)}$

is the $E W$ that would be seen if a separate spectrum could be taken of the two highly magnified images. Thus, if $E_{0}$ and $\bar{E}\left(\boldsymbol{u}_{\mathrm{c}}\right)$ are measured spectroscopically and if the fraction of light coming from the highly magnified images $f\left(\boldsymbol{u}_{\mathrm{c}}\right) \equiv \bar{A}_{2}\left(\boldsymbol{u}_{\mathrm{c}}\right) / \bar{A}\left(\boldsymbol{u}_{\mathrm{c}}\right)$ is known from the photometric light curve, then one can invert Eq. (4) to determine $\overline{E_{2}}\left(\mathbf{u}_{\mathrm{c}}\right)$,

$\frac{\overline{E_{2}}}{E_{0}}=1+\frac{\Delta}{f}, \quad \Delta \equiv \frac{\bar{E}-E_{0}}{E_{0}}, \quad f \equiv \frac{\overline{A_{2}}}{\bar{A}}$.

If we assume no blending (see Sect. 5), then $\bar{A}\left(\boldsymbol{u}_{\mathrm{c}}\right)$ is directly measured from the light curve (Fig. 1). In principle, $A_{3}$ is changing with time and so can only be known if there is a full model of the light curve. However, in general $A_{3}$ is expected to change slowly. Moreover, in the present case it is measured beginning at $\mathrm{JD}^{\prime}=1733.65$, only 2.5 hours after the last VLT measurement $\left(\mathrm{JD}^{\prime}=1733.54\right)$ and is found to be nearly constant for an hour after that. We will therefore assume that $A_{3}$ is constant throughout the crossing and will discuss possible small corrections to this approximation in Sect. 5 .

For the last VLT point, Albrow et al. (2001b) report $\bar{E}(1733.54)=0.79 \pm 0.03 \AA$, and they adopt $E_{0}=1.03 \AA$, i.e. $\Delta=-0.23 \pm 0.03$. From the EROS lightcurve (Fig. 1), $f(1733.54)=0.060 \pm 0.007$. As we will show in Sect. 4 , at this time the source center was approximately $\eta=0.963$ source radii past the caustic, so that $\overline{E_{2}}(1733.54)$ is a weighted average of the outer $4 \%$ of the source. Here $\eta$ is the perpendicular distance from the source center to the caustic in units of the source radius, and is taken to be negative when the source center is inside the caustic. Combining these facts with Eq. (6) yields

$\frac{\overline{E_{2}}(1733.54)}{E_{0}}=-2.9 \pm 0.7 \quad(\eta \simeq 0.96)$,

which is to say that the limb is in emission more strongly than the star as a whole is in absorption. As we show 
Table 1. Implications of measured $\mathrm{H} \alpha E W \mathrm{~s}$.

\begin{tabular}{ccccccccc}
\hline Telescope & $\mathrm{JD}^{\prime}$ & $\bar{E}(\AA)$ & $\sigma(\bar{E})(\AA)$ & $f$ & $\sigma(f)$ & $\eta$ & $\overline{E_{2}}(\AA)$ & $\sigma\left(\overline{E_{2}}\right)(\AA)$ \\
\hline VLT & 1730.60 & 1.06 & 0.01 & 0.846 & 0.001 & -0.595 & +1.07 & 0.01 \\
VLT & 1731.67 & 1.09 & 0.02 & 0.803 & 0.001 & +0.097 & +1.10 & 0.02 \\
VLT & 1732.66 & 0.98 & 0.01 & 0.626 & 0.001 & +0.611 & +0.95 & 0.02 \\
VLT & 1733.54 & 0.79 & 0.03 & 0.060 & 0.007 & +0.963 & -2.97 & 0.68 \\
Keck & 1731.953 & 0.944 & 0.002 & 0.773 & 0.001 & +0.256 & & \\
Keck & 1732.950 & 0.869 & 0.006 & 0.510 & 0.002 & +0.738 & & \\
\hline
\end{tabular}

in Sect. 4, this would strongly contradict the model of Albrow et al. (2001b).

We note that since the caustic is a square-root singularity (and assuming circular symmetry of the source),

$\overline{E_{2}} \simeq \frac{\int_{\eta}^{1} \mathrm{~d} r r S(r) E(r)}{\int_{\eta}^{1} \mathrm{~d} r r S(r)} \quad(1-\eta \ll 1)$,

where $r$ is normalized to the source radius. That is, $\overline{E_{2}}$ is very nearly equivalent to a simple weighted average of the $E W$ by the light coming from the entire periphery of the source.

Applying Eq. (6) to all the VLT and Keck measurements, we find,

\section{(see Table 1 above.)}

Note that since Castro et al. (2001) did not measure $E_{0}$, we cannot use Eq. (6) to evaluate $\overline{E_{2}}$, for the Keck data. One cannot simply adopt the VLT value of $E_{0}$ because the VLT and Keck data are affected by systematic differences as discussed in the third-to-last paragraph of Sect. 4. We note that D. Minniti (2001, private communication) has high-resolution VLT data for this event when it was highly (but not differentially) magnified, so it may eventually be possible to correct this shortcoming.

The results reported in Table 1 can be directly compared with results from model atmospheres, subject to some minor qualifications discussed in Sect. 5 .

\section{Predicting equivalent widths from the lightcurve}

Without loss of generality, one can write the magnification as

$A(\boldsymbol{u})=A_{3}(\boldsymbol{u})+Z(\boldsymbol{u}) \Delta u_{\perp}^{-1 / 2} \Theta\left(\Delta u_{\perp}\right)$,

where $\Delta u_{\perp}$ is the perpendicular distance from $\boldsymbol{u}$ to the caustic and $\Theta$ is a step function. As we discussed earlier, unless the source is actually probing the cusp region, it is an excellent approximation to substitute $A_{3}(\boldsymbol{u}) \rightarrow$ $A_{3}\left(\boldsymbol{u}_{\mathrm{c}}\right)$. Since the cusp approach occurred $\sim 4$ days after the caustic exit, and since $A_{3}\left(\boldsymbol{u}_{\mathrm{c}}\right)$ was observed to be almost perfectly flat for an hour after the crossing, we adopt this approximation. For closely related reasons (discussed in Sect. 5), we adopt $Z(\boldsymbol{u}) \rightarrow Z\left(\boldsymbol{u}_{\mathrm{c}}\right)$. Under these assumptions, and making use of the fact (explicitly demonstrated in Sect. 5) that the caustic curvature is small on scales of the source, the spatial averaging over the model atmosphere (required to compare theoretical predictions with the empirical results summarized in Table 1) is independent of $Z$,

$$
\begin{aligned}
& \overline{A_{2}}(\eta) \rightarrow Z\left(\boldsymbol{u}_{\mathrm{c}}\right) G(\eta), \\
& G(\eta)=\frac{1}{F_{\mathrm{s}}} \int_{\eta}^{1} \mathrm{~d} x(x-\eta)^{-1 / 2} \int_{-\sqrt{1-x^{2}}}^{\sqrt{1-x^{2}}} \mathrm{~d} y S(r), \\
& \overline{E_{2}}(\eta) \rightarrow \frac{H(\eta)}{G(\eta)}, \\
& H(\eta)=\frac{1}{F_{\mathrm{s}}} \int_{\eta}^{1} \mathrm{~d} x(x-\eta)^{-1 / 2} \int_{-\sqrt{1-x^{2}}}^{\sqrt{1-x^{2}}} \mathrm{~d} y E(r) S(r)
\end{aligned}
$$

where $x$ and $y$ are dimensionless variables and $r \equiv$ $\sqrt{x^{2}+y^{2}}$. Note that for a uniform source, $G(\eta)$ is given by e.g., Fig. 1 of Gould \& Andronov (1999).

In this section we will make the additional assumption that $A_{3}$ and $Z$ are independent of $\boldsymbol{u}_{\mathrm{c}}$,

$A_{3}\left(\boldsymbol{u}_{\mathrm{c}}\right) \rightarrow A_{3}, \quad Z\left(\boldsymbol{u}_{\mathrm{c}}\right) \rightarrow Z \quad$ (assumption).

As we discuss in Sect. 5, this assumption is less robust than others made in this paper, but is still quite reasonable for our purposes.

Albrow et al. (2001b) have presented calculations of $A(\eta)$ and $E(\eta)$ for a K3 giant atmosphere model in their Figs. 1 and 4 respectively, under the assumption that Eq. (12) is valid, for two specific choices of the pair $\left(A_{3}, Z\right)$. The resulting curves were intended only for schematic purposes, and in particular assumed that $\eta$ was a linear function of time $\left[\eta=\left(\mathrm{JD}^{\prime}-1731.8\right) / 1.9\right]$, which is known not to hold in the present case (see Eq. (13), below). However, our interest in these curves is solely that they allow us to evaluate the functions $G$ and $H$ that derive from the underlying atmosphere model. As a matter of practical computation, we must normalize $G$, and so we adopt $G_{\max }=1.40$. However, the final predictions of $E W$ are completely invariant under changes of this parameter.

We then apply these extracted functions to the EROS data. First, we measure $A_{3}=3.2294 \pm 0.0034$, where the error includes only the measurement error of the postcaustic flux (and not the baseline flux that normalizes it). This is because all predictions are invariant under changes of the assumed baseline flux. We also measure $\bar{A}_{\max }=21.02$ from the peak of the curve, and hence find $Z=\left(\bar{A}_{\max }-A_{3}\right) / G_{\max }=12.7$. Then for each observed 


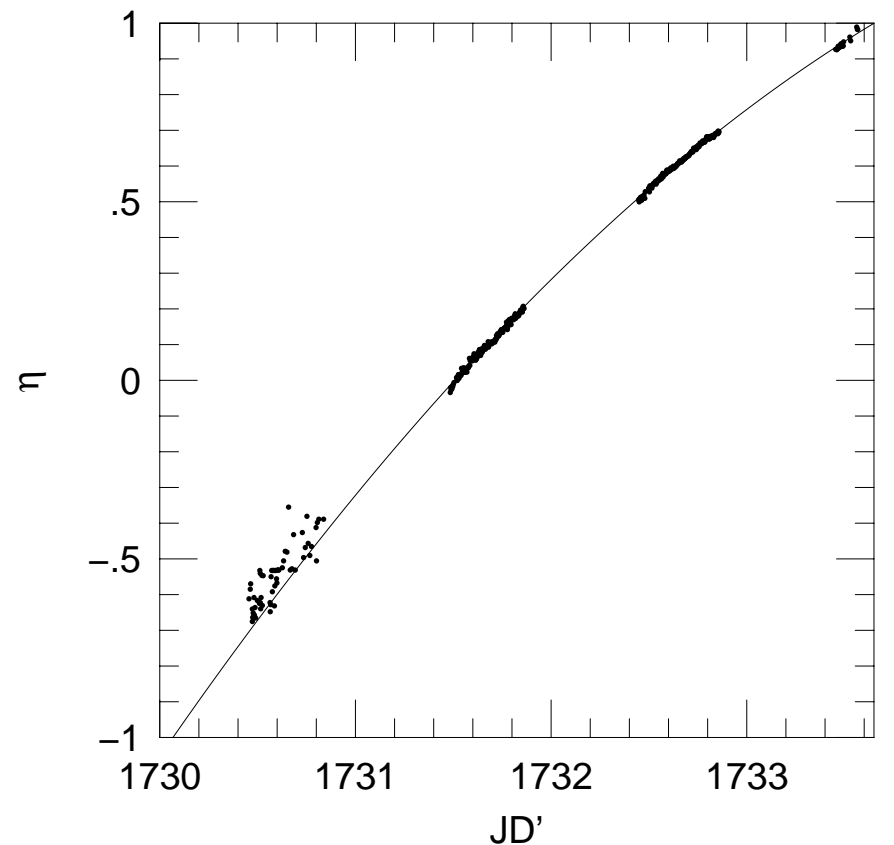

Fig. 2. Photometric determination of the perpendicular distance $\eta$ from the source center to the caustic in units of the source radius. First, we find $G(\eta)=\left(A-A_{3}\right) / Z$ where $A$ is the observed magnification (Fig. 1), $A_{3}=3.23$ is the post-caustic magnification, and $Z=\left(A_{\max }-A_{3}\right) / G_{\max }=12.7$ (see text). We then invert the stellar-profile function $G(\eta)$ from Fig. 1 of Albrow et al. (2001b) to determine $\eta$. The curve is a quadratic fit to all the points. The fit time of its endpoint $\left(\mathrm{JD}^{\prime}=1733.65\right.$ at $\eta=1$ ) occurs just before the start of the last hour of data which, from Fig. 1, is independently known to occur after the caustic exit.

magnification, $\bar{A}$, we determine $\eta=G^{-1}\left[\left(\bar{A}-A_{3}\right) / Z\right]$. Since $G^{-1}$ is double valued we must assign a branch depending on whether $\mathrm{JD}^{\prime}$ is before or after 1730.59 (the time of the observed peak of the light curve), and for points that have $\bar{A}>\bar{A}_{\max }$, due to observational error, we assign $\eta=G^{-1}\left[\left(\bar{A}_{\max }-A_{3}\right) / Z\right]$. The resulting values of $\eta$ are shown in Fig. 2. For most caustic crossings, $\eta(t)$ is reasonably approximated by a straight line, but clearly this is not so in the present case. Indeed from the cusp approach (sharp peak) in the light curve four days after the caustic crossing, it is known that the source must have exited nearly tangent to the caustic, so that the caustic curvature must play an important role. To interpolate to times when we have no data, we fit therefore the points $\eta(t)$ to a parabola whose equation is given by,

$\eta=1+0.3298\left(\mathrm{JD}^{\prime}-t_{\mathrm{cc}, \text { end }}\right)-0.0637\left(\mathrm{JD}^{\prime}-t_{\mathrm{cc}, \text { end }}\right)^{2}$,

$t_{\mathrm{cc}, \text { end }}=1733.6506$,

and whose form is shown in Fig. 2. This curve is a good fit to the data except for the first night where it deviates by approximately the scatter in the points. This shows that at least one of our assumptions is not fully satisfied: either the caustic deviates from a parabolic form, or the assumption (12) is too much of an oversimplification, or perhaps the limb-darkening model of
Albrow et al. (2001b) does not perfectly reproduce $G(\eta)$ for the EROS $V_{\mathrm{E}}$ band. Whatever the cause of the deviation, the fact that it is more pronounced on the first night is simply due to the fact that the errors in $\eta$ are larger. This in turn can be traced to the fact that this night covers the peak of the curve (see Fig. 1 ), where $\mathrm{d} G / \mathrm{d} \eta \sim 0$. The error in the estimate of $\eta$ for each measured point is given by $\sigma(\eta)=\sigma\left(F_{V}\right) /\left(F_{V}\right.$, baseline $\left.Z|\mathrm{~d} G / \mathrm{d} \eta|\right)$. These larger errors are reflected in the larger scatter of the points on the first night in Fig. 2. The errors are not displayed in Fig. 2 in order to avoid clutter, but are shown in a different projection in Sect. 5 .

Note that the error on the time of the end of the caustic crossing $(\eta=1)$, derived from the fit leading to Eq. (13), is very small, $t_{\mathrm{cc} \text {, end }}=1733.6506 \pm 0.0048$. However, the actual error is dominated by the assumption that the limbdarkening model of the curve shown in Fig. 1 (ultimately derived from Fig. 1 of Albrow et al. 2001b) is correct, which it almost certainly is not at this level. Nevertheless, $t_{\mathrm{cc} \text {, end }}$ cannot be much after this best-fit value because the observed light curve is flat (or very slightly rising) beginning at this time.

Finally, we predict the $E W, \bar{E}(t)$, using the values of $\eta(t)$ found from Eq. (13), the tabulated functions $G(\eta)$ and $H(\eta)$, the measured values of $\bar{A}(t)$ and $A_{3}$, and Eqs. (4) and (11). The result is shown in Fig. 3.

As can be seen from Table 1, the VLT EWs are about $0.1 \AA$ larger than the corresponding Keck $E W$ s, even taking account of the fact that the observations were a few hours earlier. One possible explanation for this discrepancy is that the low-resolution VLT spectra were affected by blended lines that were removed from the Keck HIRES spectra. From Fig. 2 of Castro et al. (2000), this indeed appears plausible. However, the offset could also be due to differences in the way the $E W \mathrm{~s}$ were measured, which is not explained in detail in either paper. Whatever the exact cause, it is clear that there are systematic differences between the two series of measurements. For purposes of displaying our results in Fig. 3, we therefore assume that $0.1 \AA$ of the VLT EW s are due to blends. Hence, we reduce all of them by this amount and correspondingly rescale the Albrow et al. (2001b) model downward by $10 \%$. There remains the freedom to adjust the relative offsets between the Keck and VLT points (because our 10\% VLT blending estimate is only a guess), and to adjust the overall scale of the curve (because this is set by $E_{0}$ which is measured from the VLT pre-caustic spectrum - but with a large error bar).

The uncertainties in $\overline{E_{2}}$ reported in Eq. (7) and in Table 1 reflect the photometric and EW measurement errors at the individual times, but not errors in $E_{0}$ or a possible systematic offset in all $E W$ s (induced, e.g., by blending), either of which would affect all estimates of $\frac{\overline{E_{2}}}{E_{2}}$ together. A systematic offset of $0.1 \AA$ due e.g., to blending would not change at all the difference $\overline{E_{2}}-E_{0}=E_{0} \Delta / f$ see Eq. (6). That is, all the inferred limb $E W \mathrm{~s}$ would move down by the same amount as $E_{0}$. A systematic error $\delta E_{0}$ of $0.05 \AA$ in the pre-crossing measurement of $E_{0}$ 


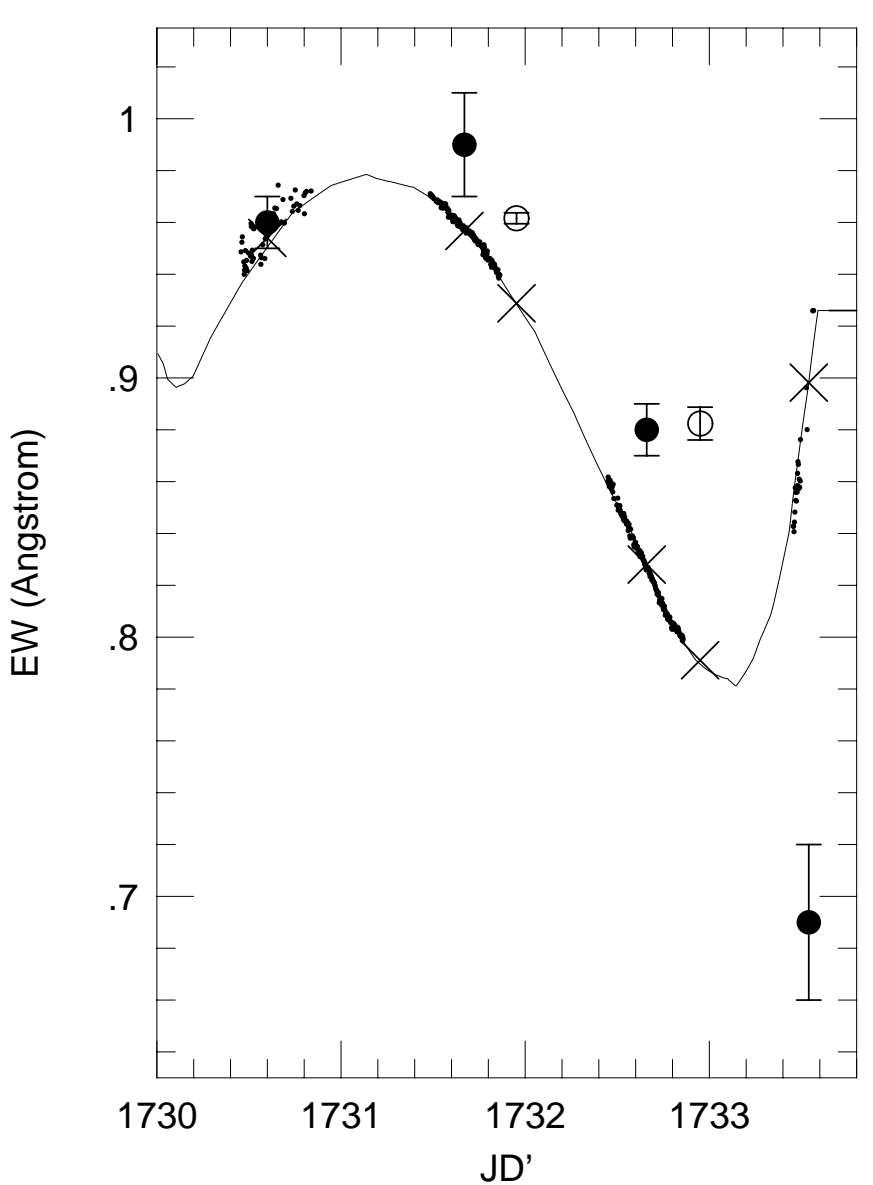

Fig. 3. Measured and predicted $\mathrm{H} \alpha$ equivalent widths $(E W \mathrm{~s})$. Small points show the predictions from the photometric data. For each measured magnification (Fig. 1), the source-caustic separation $\eta$ is determined (Fig. 2), and so also the $E W$ of the two highly magnified images inside the caustic, $\overline{E_{2}}=$ $H(\eta) / G(\eta)$. Here $G$ and $H$ are functions extracted from the atmosphere model of Albrow et al. (2001b). See text. The predicted $E W$ is then the weighted average of $\overline{E_{2}}$ and $E_{0}$, the $E W$ of the unmagnified source. The curve results from applying the same calculation to the curves in Figs. 1 and 2. Solid points are measured $E W \mathrm{~s}$ from VLT FORS1 low-resolution data of Albrow et al. (2001b) and open points are from HIRES KECK data of Castro et al. (2001). Crosses show where these points should be if they agreed with the model. The VLT points and the corresponding model have been rescaled assuming a $10 \%$ blend from a neighboring line. This is plausible based in Fig. 2 from Castro et al. (2001) and brings the VLT points approximately into line with the Keck points. However, no amount of rescaling would bring the atmospheric model into agreement with all the data points.

(equal to the measurement error quoted by Albrow et al. $2001 b)$ would cause changes in the limb $E W$ estimates of $\delta\left(\overline{E_{2}} / E_{0}\right)=-\left(\delta E_{0} / E_{0}\right) \bar{E} / f E_{0} \sim \pm 0.05 \bar{E} / f E_{0}$. For the last VLT measurement this is $\sim 0.65$, almost as large as the statistical error. However, the result of Eq. (7) would still be significant at the $3 \sigma$ level. Moreover, any model that predicts the $E W$ as a function of time will be much more strongly constrained by the $E W$ measurement on HJD $^{\prime}=1730.6$ (with its error of $0.01 \AA$ ) than it will by the pre-caustic measurement (with its $0.05 \AA$ error). Hence, the true systematic error in $\overline{E_{2}} / E_{0}$ is more like $0.13 \AA$, i.e., much less than the statistical error.

As we have noted above, there is some flexibility in the relative alignment of the model and data in Fig. 3. However, there is no amount of adjustment that can reconcile the points with the curve. The point with the largest deviation between observed and predicted $E W$ is the last VLT spectrum. In the previous section we showed that the model could predict such a low $E W$ only if the outer $4 \%$ of the source were strongly in emission. By contrast, the model of Albrow et al. (2001b) predicts that the outer $4 \%$ will have substantial (albeit reduced) absorption: $\overline{E_{2}}(0.963)=H(0.963) / G(0.963)=0.0105 \AA / 0.0184=$ $0.57 \AA$ (with no correction for line blends).

\section{Examination of assumptions}

We have used EROS lightcurve data to facilitate the comparison of spectra taken during the caustic crossing of EROS-BLG-2000-5 with an atmosphere model of the K3 source. An alternative approach would be to fit the lightcurve data to a lens model and then apply the lens model directly to the atmosphere model to make predictions about the spectra. The latter approach would appear to be more secure but, unfortunately, to date there are no published models of this event, and the event may prove difficult to model. Thus, since our approach is the only one available at present, it will be worthwhile to go through the assumptions that were introduced and see what order of errors they induce. This review can also serve as a checklist if our method is applied to future events.

We assumed no blending. There are three lines of evidence that this is a good assumption. First, the lensed source is a bright giant, so it is fairly improbable that it would be seriously blended. Second, there is no evidence for blended light in the spectrum of Castro et al. (2001) as there would be if there were a bright blend offset by at least $15 \mathrm{~km} \mathrm{~s}^{-1}$ in radial velocity from the source. Third, the event is nearly achromatic (see Fig. 4). This means that either the blend is very weak in which case it has little effect, or it has virtually the same color as the source, and so very likely has the same $\mathrm{H} \alpha E W$ as well. To the extent that this is the case, the predicted $E W$ s are exactly the same as in the unblended case.

Next, we assumed that the non-divergent magnification field $A_{3}(\boldsymbol{u})$ could be replaced by its value at the center of the source, $A_{3}\left(\boldsymbol{u}_{\mathrm{c}}\right)$. This is valid to the extent that on scales of the source size, the $A_{3}$ field is equal to a constant plus a gradient. The only way this could fail to hold would be if the source were probing the neighborhood of a cusp. It is clear that the source is approaching a cusp, because the lightcurve betrays a characteristic cusp-approach behavior 4 days after the caustic exit. However, in the hour after the caustic exit, the light curve is nearly flat, indicating that this cusp is not yet significanly affecting the magnification pattern at the time of the exit. 


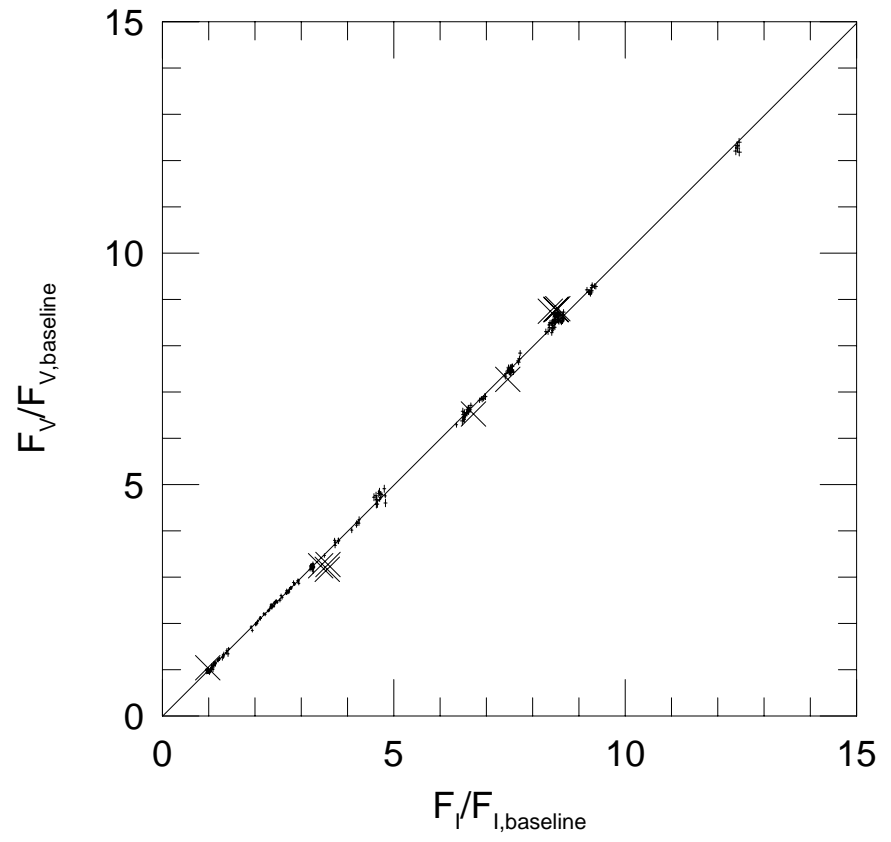

Fig. 4. Comparison of fluxes in two bands $V_{\mathrm{E}}$ and $I_{\mathrm{E}}$ for all points on the EROS light curve except during the two caustic crossings and the cusp approach (when differential magnification could induce color changes). The regression (solid line) passes extremely close to the origin, showing that either the event is not significantly blended or if it is, the color of the blend is extremely close to that of the source. The large crosses show the 10 outlier points (out of 250) that were excluded from fit.

Third, we assumed that $A_{3}$ would be constant throughout the crossing. The measured logarithmic slope of $A_{3}$ immediately after the crossing is $\mathrm{d} \ln A_{3} / \mathrm{d} t=(0.11 \pm$ $0.08)$ day $^{-1}$, so that at the time of the last spectrum measurement (0.11 days before the end of the crossing), $A_{3}$ was within a percent or two of the final value. A $1 \%$ change in $A_{3}$ leads to a change of $\sim-17 \%$ in $f$ and so of $\sim-0.5$ in Eq. (7). This is not negligible, but it would not qualitatively affect the conclusion that the outer $4 \%$ of the source is in emission. Plausibly, at much earlier times $A_{3}$ could have differed from its final value by $\sim 10 \%$, but as these times such a change would have a very small impact on the conclusions simply because $A_{3}$ does not dominate the total flux. For example, suppose this had been the case at $\mathrm{JD}^{\prime}=1732.66$. Then $f$ would have fallen from 0.626 to 0.588 , which in turn would change $\overline{E_{2}}$ by only $0.005 \AA$. These are all the assumptions needed through Sect. 3.

In Sect. 4, we went on to make three additional assumptions. The first was that on the scale of the source the caustic could be approximated as a straight line. From the measurements of the first crossing, the source is known to cross its own diameter in $\lesssim 1$ day. If we interpret the quadratic behavior of Eq. (13) as arising from the curvature of the caustic, then over a diameter, the caustic deviates from a straight line by $\sim 8 \times 10^{-3}$ of a source radius, certainly small enough to ignore.

Second, we assumed that at any instant $Z(\boldsymbol{u})$ could be replaced by its value at its center $Z\left(\boldsymbol{u}_{\mathrm{c}}\right)$. Or equivalently, that we are in the square-root singularity regime and the coefficient of this regime varies at most linearly over a source diameter. From Table 1 and the adopted value of $Z=12.7$, one sees that that at the trailing limb of the source for the first measured point, $A_{2}(\boldsymbol{u})=10$, which is normally well into the square-root singularity. Since the source diameter is $\$ 1$ day and at the exit it is still 4 days from the post-caustic peak in the light curve due to a cusp approach, the assumption that quadratic variation of $Z$ is not significant on the scale of the source size appears very reasonable, but by no means proven.

Third, we assumed that $A_{3}$ and $Z$ remain constant throughout the crossing. We discussed above why this is a reasonable assumption for $A_{3}$. The argument regarding $Z$ is similar. Suppose that $Z$ in fact fell linearly by $20 \%$ from the time of the peak until the end of the crossing (and suppose $A_{3}$ remained constant). The product $G(\eta) Z=$ $\overline{A_{2}}$ is then an observable and so remains fixed. Thus, at the last measurement, $Z$ would be $19 \%$ lower, so $G(\eta)$ would actually be $24 \%$ higher than we have allowed. This would drive $\eta$ down from 0.963 to 0.956 , but it would affect the $E W$ prediction shown in Fig. 3 by well under $1 \%$. Hence, while it would be better not to have to make this approximation, the errors it induces are extremely small compared to the level of disagreement between the models and the data in Fig. 3.

To directly probe the scale of the systematic errors introduced by our approximations, we conduct the following test. Instead of adopting the $G_{\mathrm{P}}(\eta)$ derived from the atmosphere model of Albrow et al. (2001b), we directly fit for $G_{V}(\eta ; c)$ from the observed $V_{\mathrm{E}}$ magnification, $A_{V}(t)$, using the 6-parameter functional form,

$A_{V}(t)=A_{3}+Z G_{V}[\eta(t) ; c]$

where $c$ is a linear limb-darkening coefficient, and $\eta$ is a (3-parameter) quadratic function of time as in Eq. (13). We find $c=0.644 \pm 0.032, t_{\mathrm{cc} \text {, end }}=1733.6280$, and linear and quadratic coefficients 0.3421 and -0.0596 , respectively. The resulting curve $\eta(t)$ (Fig. 5) is very similar to the corresponding curve (Fig. 2) for $G_{\mathrm{P}}$. Moreover, when we evaluate $\eta$ using $G_{V}$ instead of $G_{\mathrm{P}}$, we find that the predicted $E W$ s are almost exactly the same as those shown in Fig. 3: the changes are an order of magnitude smaller than the error bars on the VLT and Keck data points.

Figure 6 shows the photometric residuals of the fit during the first night $\left(\mathrm{JD}^{\prime} \sim 1730.6\right)$, for which the residuals in terms of $\eta$ seen in Fig. 5 are most pronounced. Typically, these residuals are extremely small, $<0.005 \mathrm{mag}$, and are of order the individual errors. However, taken together they show a clear trend which is highly significant: the observed light curve peaks earlier than the model. The offset is about 0.1 day, or $\Delta \eta \sim 1 / 15$. This level of discrepancy induces an offset in the predicted $E W$ on the first night of about $1 / 2$ the statistical error of the $E W$ measurement (see Fig. 3). However, since the model is only used to interpolate to times when there are no photometric data, and since the model and data are in excellent agreement in the neighborhoods of the Keck points (which are the 


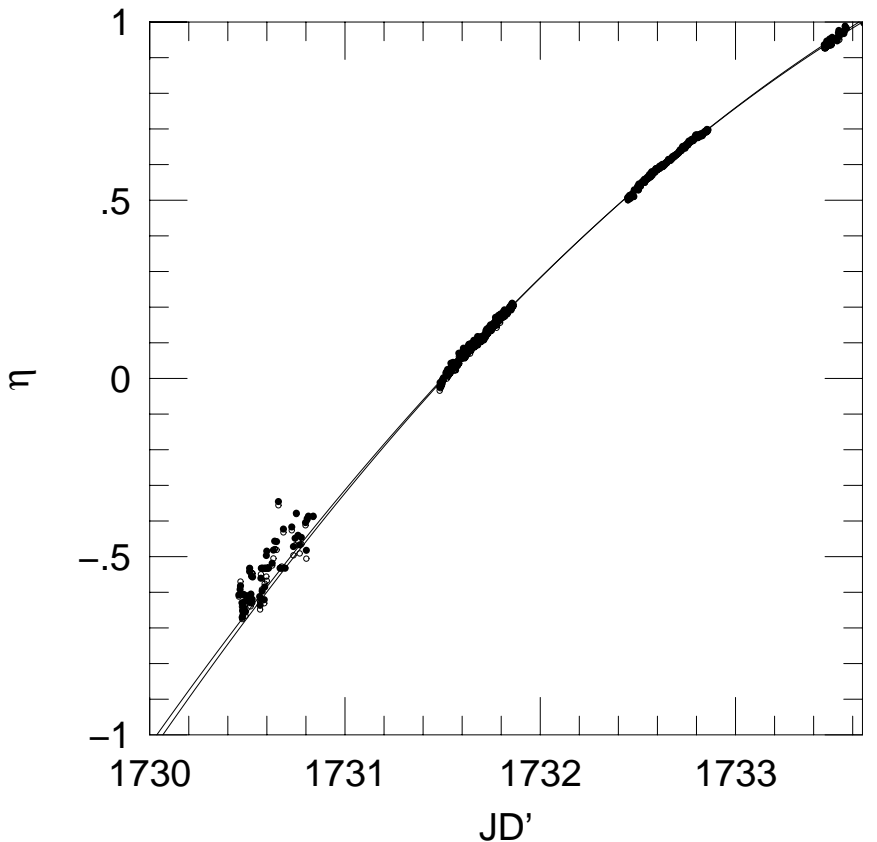

Fig. 5. Filled points and upper curve: photometric determination of the perpendicular distance $\eta$ from the source center to the caustic in units of the source radius, obtained by directly fitting the EROS $V_{\mathrm{E}}$ data to a limb-darkened profile function $G_{V}(\eta ; c)$, where the linear limb-darkening parameter $c$ is treated as a free parameter and is found to be $c=0.644 \pm 0.032$. Open points and lower curve: same as Fig. 2, which was based on the stellar profile function $G_{\mathrm{P}}(\eta)$ taken from Albrow et al. (2001b). The a priori model $G_{\mathrm{P}}(\eta)$ yields almost exactly the same curve as the limb-darkening fit $G_{V}(\eta ; c)$.

only ones where there is no coincident photometry), this discrepancy has no practical impact.

Parenthetically, we note that while one might be tempted to take the good agreement shown in Fig. 5 as supporting the believability of the derived limb-darkening coefficient, in fact this determination must be viewed skeptically. Any change in $A_{3}$ or $Z$ during the very long crossing, as well as any deviation of the caustic from a purely parabolic form, will be subsumed in the limb-darkening coefficient. Hence, a valid measurement of $c$ can be obtained only in conjunction with a complete determination of the geometry of the binary lens. The main implication of this fit is that, while the limb-darkening coefficient may be significantly affected by these various deviations from a simple model, the predictions of equivalent widths are not.

We conclude that all the assumptions we have made are quite reasonable, at least for the present case. Future applications of this method will have to be carefully justified based on an analysis of the event that is parallel to the one given here.

\section{Color of the limb}

Essentially the same method presented above can be used to find the color of the two highly magnified (caustic) images. Recall from Eq. (8) that for $1-\eta \ll 1$, this is

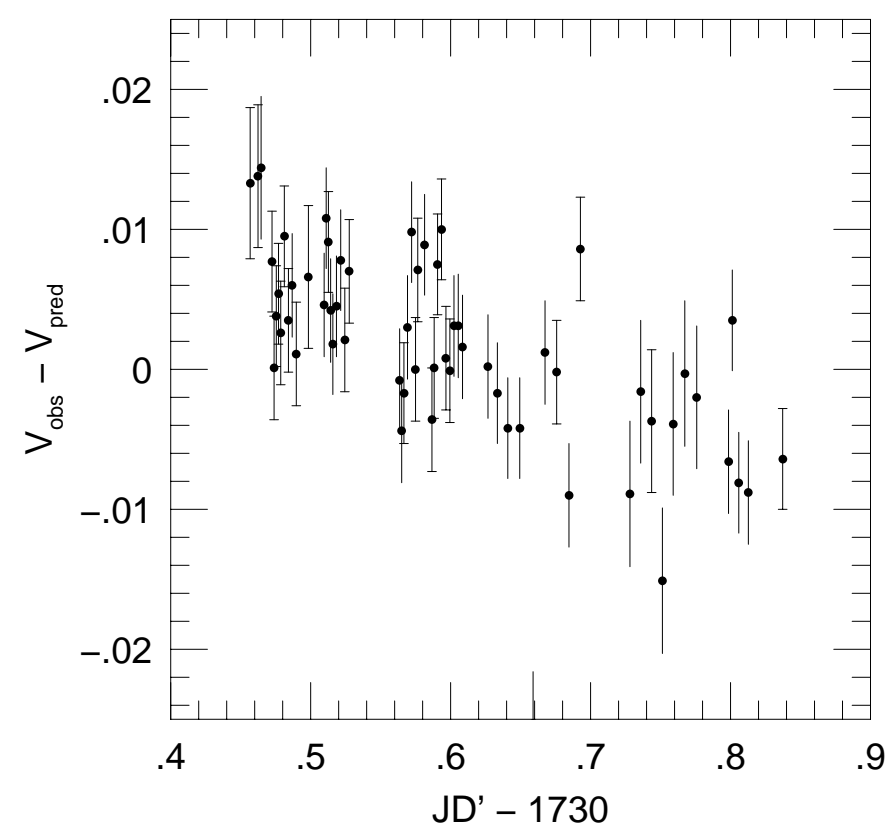

Fig. 6. Photometric $\left(V_{\mathrm{E}}\right)$ residuals on the first night of the caustic crossing, relative to the linear limb-darkening fit, with a parabolic $\eta(t)$ shown in Fig. 5 . The individual residuals are extremely small, but the trend is highly significant, showing that the real light curve peaks earlier than the model by about 0.1 days.

very nearly equivalent to the color of the annulus of the source $1-\eta<r<1$. The difference in the color of the two highly magnified images relative to the unmagnified source is given by,

$$
\begin{aligned}
\Delta\left(V_{\mathrm{E}}-I_{\mathrm{E}}\right) & \equiv\left(V_{\mathrm{E}}-I_{\mathrm{E}}\right)_{2}-\left(V_{\mathrm{E}}-I_{\mathrm{E}}\right)_{\text {source }} \\
& =2.5 \log \left(\frac{F_{I, 2}}{F_{V, 2}} \frac{\mathrm{d} F_{V}}{\mathrm{~d} F_{I}}\right)
\end{aligned}
$$

where $F_{I, 2}$ and $F_{V, 2}$ are the fluxes due to the two caustic images, and $\mathrm{d} F_{V} / \mathrm{d} F_{I}$ is the slope shown (normalized to the baselines) in Fig. 4. Since this figure is restricted to points away from the caustics and cusps where the source is not differentially magnified, $-2.5 \log \left(\mathrm{d} F_{V} / \mathrm{d} F_{I}\right)$ is the color of the source. Under the assumption (12),

$F_{V, 2}=F_{V}-F_{V, \text { end }}$,

where $F_{V}$ is the observed $V_{\mathrm{E}}$ flux at a given time and $F_{V \text {, end }}$ is the flux just after the end of the caustic crossing (and similarly for $I_{\mathrm{E}}$ ). Note that Eqs. (15) and (16) are valid independent of whether the source is blended, and that when combined they can be evaluated from directly observed quantities without any modeling. The only assumption is that $A_{3}$ does not change significantly between the time of the measurement and the end of the caustic crossing.

Figure 7 shows the result of applying Eqs. (15) and (16) to the EROS data. The time coordinate has been changed to source position relative to the caustic $(\eta)$ using Eq. (13). The color difference is small to negligible on the first two nights $\left[\Delta\left(V_{\mathrm{E}}-I_{\mathrm{E}}\right)=0.0006 \pm 0.0006\right.$ and $-0.0015 \pm 0.0006$ 


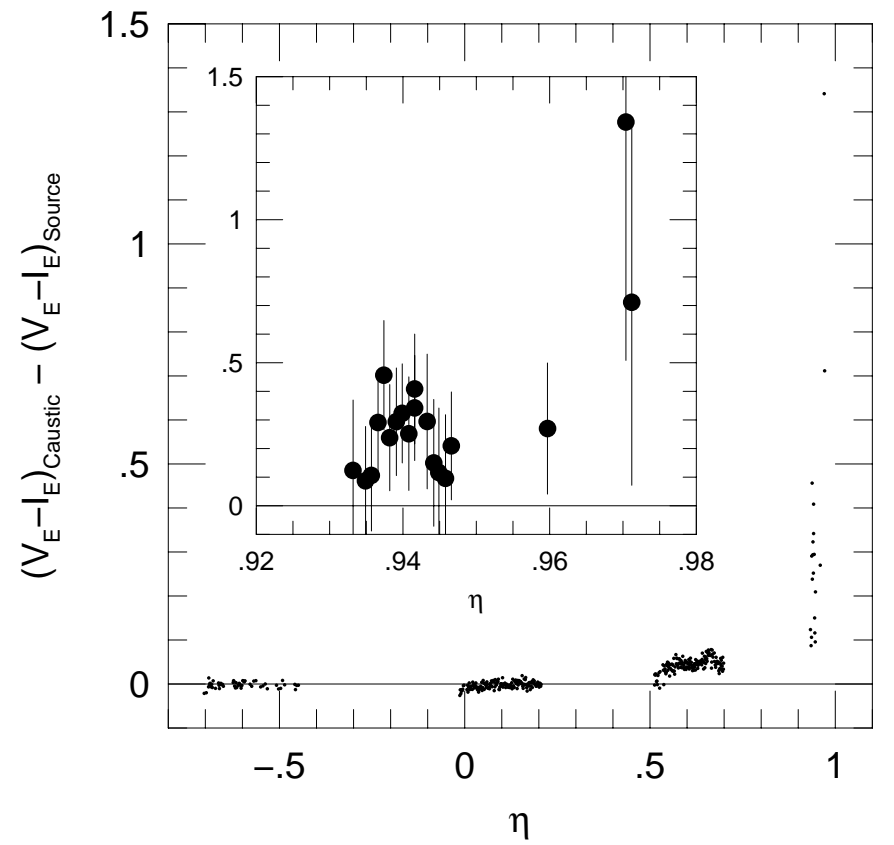

Fig. 7. Difference in $\left(V_{\mathrm{E}}-I_{\mathrm{E}}\right)$ color between the two highlymagnified caustic images and the source as a whole, as a function of $\eta$, the separation of the source center from the caustic in units of the source radius. The color shift is extremely small and only marginally significant on the first two nights, but rises to $0.046 \pm 0.001$ on the third night and $0.27 \pm 0.02$ on the last, when the caustic images are equivalent to looking at the outer $5.5 \%$ of the source. Since the source is a K3 giant, this limb has the color of a M0 giant.

for $\eta \sim-0.6$ and $\eta \sim 0.1$ respectively] reflecting the fact that the caustic images receive significant contributions from a range of radii at these positions (Gaudi \& Gould 1999). On the third night, the caustic images are significantly redder, $\Delta\left(V_{\mathrm{E}}-I_{\mathrm{E}}\right)=0.046 \pm 0.001$ at $\eta \sim 0.6$. Here, the analog of Eq. (8) approximately holds, so that the color of the caustic images is the same as that of the outer $1-\eta \sim 40 \%$ of the source (by radius). Finally, on the last night, we find $\Delta\left(V_{\mathrm{E}}-I_{\mathrm{E}}\right)=0.27 \pm 0.02$ at $\eta \sim 0.945$, equivalent to an annulus comprising the outer $5.5 \%$ of the source. This is roughly equivalent to $\Delta(V-I) \sim 0.37$ in standard Johnson/Cousins bands. Hence, assuming that the K3 source has a typical $(V-I)_{0} \sim 1.36$ color (Bessell $\&$ Brett 1988), the limb has $(V-I)_{0} \sim 1.73$, i.e. the color of a M0 giant.

\section{Probing the chromosphere?}

The color (and thus temperature) difference of the source periphery relative to the source as a whole $(\Delta(V-I) \sim$ $0.37)$ is modest compared to the dramatic shift from $\mathrm{H} \alpha$ absorption to emission implied by Eq. (7). That is, M0 stars absorb rather than emit $\mathrm{H} \alpha$. Hence, to explain this shift it would appear necessary to invoke some additional structure in the source atmosphere. The chromosphere presents itself as an interesting candidate. We therefore evaluate what strength of chromospheric emission is required to account for the sharp decline in $E W$ reported by Albrow et al. (2001b) on the last night.

Seen in projection above the limb of the photosphere, the solar chromosphere emits strongly in $\mathrm{H} \alpha$ and is essentially black in the neighboring continuum. If we assume that the same is true of the source of EROS-BLG-2000-5, then Eq. (4) can be generalized to

$$
\bar{E}\left(\boldsymbol{u}_{\mathrm{c}}\right)=\frac{A_{3}\left(\boldsymbol{u}_{\mathrm{c}}\right) E_{0}+\overline{A_{2}}\left(\boldsymbol{u}_{\mathrm{c}}\right) \overline{E_{2}}\left(\boldsymbol{u}_{\mathrm{c}}\right)-\overline{A_{2, \text { chrom }}}\left(\boldsymbol{u}_{\mathrm{c}}\right) E_{0} X}{A_{3}\left(\boldsymbol{u}_{\mathrm{c}}\right)+\overline{A_{2}}\left(\boldsymbol{u}_{\mathrm{c}}\right)},
$$

where $E_{0}$ still represents the $E W$ of the unmagnified source, including the effect of $\mathrm{H}_{\alpha}$ chromospheric emission, $X$ is the ratio of this chromospheric emission to the algebraic sum of the $\mathrm{H}_{\alpha}$ absorption by the stellar atmosphere and of the chromospheric emission, and $\overline{A_{2, \text { chrom }}}\left(\boldsymbol{u}_{\mathrm{c}}\right)$ is the magnification of the chromosphere by the two caustic images. Note that $\overline{A_{2, \text { chrom }}}$ is the ratio of the $\mathrm{H}_{\alpha}$ chromosphere photon flux in the two caustic images to the $\mathrm{H}_{\alpha}$ chromosphere photon flux normally received in the absence of magnification, and so is not exactly analogous to $\overline{A_{2}}$, which is normalized to the number of photons normally received from the source as a whole.

It is straightforward to show that in the limit $\eta \rightarrow 1$,

$\overline{A_{2, \text { chrom }}} \rightarrow \frac{Z_{*}}{\sqrt{2}} \quad(\eta \rightarrow 1)$

See for example Fig. 1 from Castro et al. (2001). Here, $Z_{*}$ is the magnification due to the two caustic images of a point source lying exactly one source radius inside the caustic. We will adopt $Z_{*}=12.7$, which is the value measured in Sect. 4. As discussed in Sect. 5, $Z$ is actually a function of $\boldsymbol{u}_{\mathrm{c}}$ and so in principle could be different at the peak of the light curve $(\eta \sim-0.5)$ where it is measured, from its value at $\eta=0.963$. We therefore assign this parameter an error of $20 \%$. (In addition, an error could be introduced if $G_{\max }$ for the actual limb-darkened profile differed from the one adopted. However, for a reasonable range of limb-darkening profiles, this uncertainty is $<2 \%$.) Solving Eq. (17) for $X$ and substituting in values for the various parameters, we find

$X=\sqrt{2} \frac{A_{3}}{Z_{*}}\left[\left(1-\frac{\bar{E}}{E_{0}}\right)-\frac{\overline{A_{2}}}{A_{3}}\left(\frac{\bar{E}}{E_{0}}-\frac{\overline{E_{2}}}{E_{0}}\right)\right]=0.08 \pm 0.02$,

where we have taken $\overline{E_{2}} / E_{0}=0.57$ as evaluated in Sect. 4 based on the Albrow et al. (2001b) model. Note that since the ratio of the two terms within the brackets is $\sim 0.05$, uncertainties in the estimate of $\overline{E_{2}} / E_{0}$ are not likely to significantly impact the final result.

This value of $X$ is about two orders of magnitude higher than the corresponding quantity for the Sun, but giant stars may have a more significant chromosphere than the Sun. Alternatively, the errors in the $\mathrm{H} \alpha$ measurement may have been underestimated. In any event, this estimate of $X$ can be directly compared with chromosphere models. 
Acknowledgements. We are grateful to D. Lacroix and the technical staff at the Observatoire de Haute Provence and to A. Baranne for their help with the MARLY telescope. We are also grateful for the support given to our project by the technical staff at ESO, La Silla. We thank J. F. Lecointe and A. Gomes for assistance with the online computing. We thank G. Newsom for useful discussions on the solar chromosphere. Work by AG was supported by NSF grant AST 97-27520 and by a grant from Le Centre Français pour L'Accueil et Les Échanges Internationaux.

\section{References}

Afonso, C., et al. 2000, ApJ, 532, 340

Alard, C. 2000, A\&AS, 144, 363

Albrow, M., et al. 1999, ApJ, 522, 1011
Albrow, M., et al. 2000, ApJ, 534, 894

Albrow, M., et al. 2001a, ApJ, 549, 759

Albrow, M., et al. 2001b, ApJ, 550, L173

Alcock, C., et al. 1997, ApJ, 491, 436

Ansari, R., et al. 1996, Vistas Astron., 40, 519

Bessell, M. S., \& Brett, J. M. 1988, PASP, 100, 1134

Castro, S. M., Pogge, R. W, Rich, R. M., DePoy, D. L., \& Gould, A. 2001, ApJ, 548, L197

Gaudi, B. S., \& Gould, A. 1999, ApJ, 513, 619

Gould, A., \& Andronov, N. 1999, ApJ, 516, 236

Heyrovský, D., Sasselov, D., \& Loeb, A. 2001, ApJ, 543, 406 Landolt, A. U. 1992, AJ, 104, 340

Lennon, D. J., Mao, S., Fuhrmann, K., \& Gehren, T. 1996, ApJ, 471, L23

Paczynski, B., et al. 1999, Acta Astron., 49, 319

Valls-Gabaud, D. 1998, MNRAS, 294, 747 